\title{
The role of left prefrontal transcranial magnetic stimulation in episodic migraine prophylaxis
}

\author{
Randa Amin ${ }^{1}$, Tamer Emara ${ }^{1 *}$, Samia Ashour ${ }^{1}$, Mahmoud Hemeda', Nahed Salah Eldin', Salma Hamed', \\ Sara Shouman ${ }^{2}$ (D) and Mohamed Shouman ${ }^{3}$
}

\begin{abstract}
Objective: The aim of the study was to examine the prophylactic role of repetitive transcranial magnetic stimulation (rTMS) on the frequency, and severity of migraine attacks in episodic migraineurs who failed medical treatment.

Methods: A randomized double-blinded placebo-controlled study was designed to assess the effect of $5 \mathrm{~Hz}$ rTMS applied over the left dorsolateral prefrontal cortex (LDLPFC) in 33 migraineurs. Patients were followed up for 1 month before receiving rTMS, and for another month after the sessions by a headache diary. The primary outcome measure was the achievement of 50\% reduction in the number of migraine attacks. Secondary outcome measures included migraine days, assessment of migraine attack severity, disability by HIT-6, and side-effects to the procedure.
\end{abstract}

Results: The study revealed that $69.2 \%$ of the active treatment group achieved $50 \%$ or more reduction in the number of migraine attacks versus $25 \%$ of cases in the control group $(p=0.02)$. The absolute number of migraine attacks was reduced by 3.1 vs 1.5 in the active and control group, respectively. The number of cases with severe HIT-6 scores was reduced by $46.2 \%$ in active treatment group versus a $7.1 \%$ reduction in the control group $(p=$ 0.02).

Conclusion: High-frequency rTMS applied to LDLPFC can reduce the number of migraine attacks by $50 \%$ or more in almost $70 \%$ of a sample of episodic migraineurs with a concomitant decrease in functional disability.

Trial registration: ClinicalTrials.gov, Identifier: NCT04031781. Registered 23 July 2019—retrospectively registered at https://linicaltrials.gov/ct2/show/NCT04031781?term=Migraine+Prophylaxis\&recrs=ce\&type=Intr\&cond=Migraine\&rank=9

\section{Introduction}

The incidence of a new migraine diagnosis is increasing [1]. Migraine is classified into an episodic and a chronic form based on the frequency of migraine attacks per month. The two forms despite representing a spectrum are currently regarded as different entities. Episodic migraine, which is considered as the milder form, has an annual conversion rate of $2.5 \%$ to chronic migraine [2].

Causes of conversion include non-modifiable factors related to the course of the disease, and modifiable

\footnotetext{
*Correspondence: tamer_emara@med.asu.edu.eg

${ }^{1}$ Neurrology Department, Faculty of medicine, Ain Shams University, Abbasia, Cairo, Egypt

Full list of author information is available at the end of the article
}

causes like medication overuse, increased body mass index, and the presence of depressive symptoms [3]. These factors are inappropriately managed in many cases because of a lack of specialist attention and hence delayed the start of specific prophylactic measures.

Trials to improve the treatment outcomes of episodic migraines would therefore, decrease the overall disease burden. This is especially true given the limited effectiveness and poor tolerability of the currently available prophylactic medications [4]. Single-pulse and repetitive TMS have been recently tried in the acute and prophylactic management of migraine, respectively [5]. The results of the acute management trials were encouraging 
[6]. However, most of the work done on the prophylactic role of rTMS targeted chronic migraineurs with conflicting results, apart from one study that recruited episodic and chronic migraineurs and reported positive results [7-9].

In an effort towards resolving this conflict and clarifying the best responders to rTMS, we decided to work only on cases with episodic migraine. So, this placebocontrolled pilot study examined the prophylactic role of LDLPFC high-frequency rTMS in a group of episodic migraineurs.

\section{Methods}

\section{Patient selection}

Thirty-three consecutive cases diagnosed as episodic migraine with or without aura according to the ICHD third edition, with a headache frequency of 4-14 per month for the last 6 months and older than 16 years were recruited from the neurology and headache clinics in Ain Shams University Hospitals. All patients had either an unsatisfactory response, declined or were intolerant to at least two prophylactic medications [10].

Patients with past or family history of seizures, other chronic pain disorders, severe depression (Beck Depression Inventory of 30 or more), other significant neurologic or psychiatric diagnosis including substance misuse, metal implants in the head, pregnant or breastfeeding ladies, or prior experience with TMS were excluded.

The patients were randomized through dark sealed envelopes into an active group $(n=14)$ or a sham group $(n$ $=19$ ). The baseline and follow up assessments were done by two experienced neurologists. Both neurologists as well as the patients were blinded to the type of stimulation. Two well-trained technicians delivered the sessions. They were not involved in patient assessments, follow-up, or data analysis. Four patients dropped out during the treatment phase and follow-up (three from the control group and one from the active treatment group) due to lack of response and travel.

\section{Determining cortical excitability}

Magnetic stimulation was administered with a Dantec MagLite stimulator (Dantec Corporation, Skovlunde, Denmark), using a figure-of-eight (MC-B70) coil. The handle of the coil was placed on the left hemisphere posterior to the midline at a $45^{\circ}$ angle then moved forwards at $1-\mathrm{cm}$ steps. Once the hot spot was identified, the motor threshold (MT) was determined as the lowest stimulation intensity that produces a visible abduction of the right thumb. This procedure was repeated before every rTMS session.

LDLPFC was localized five centimeters anterior to the hot spot [11].

\section{Treatments}

The active treatment group received 5 rTMS sessions, delivered over one week. Each rTMS session consisted of a single train of 900 total pulses over 3 min duration given at $5-\mathrm{Hz}$ frequency and 100\% motor threshold intensity. Sham rTMS was given with the same stimulation frequency at a fixed intensity of $50 \%$ of the machine output. The coil was held perpendicular to the brain surface over the left DLPFC site. There was no change in the treatments received by the patients for 2 months before active enrollment and during the study period.

\section{Assessments}

Patients were followed up for one month before receiving rTMS, and for another month after the sessions by a headache diary. The primary outcome measure was defined as the reduction of migraine attack frequency by at least 50\% after rTMS sessions. Secondary outcome measures included migraine days, number of abortive pills used per month, the intensity of pain that was assessed using the 0 to 10 Numeric Pain Rating Scale (NPRS), and the duration of migraine attacks. Severe migraine days were defined as those with NPRS more than 7. For all the aforementioned migraine severity parameters, a 50\% reduction was defined as the treatment target and was calculated together with the absolute reduction or change is a certain parameter. Functional disability was measured using the HIT- 6 score. Severe HIT- 6 was defined as a score of 60 or more [12]. A clinically significant change in the HIT-6 was defined as a change of 5 points or more [13].

\section{Statistical methods}

The data was analyzed with SPSS (Statistical Package for Social Science) version 20. Descriptive statistics were displayed as mean \pm standard deviation (SD) for continuous data and case count and percentage for categorical data. The statistical tests used for inter-group comparisons were the chi-square and the independent samples $t$ test. Within-group comparisons were done using the paired $t$ test. The level of statistical significance $p$ value was set at 0.05 .

\section{Data availability}

Anonymized data of patients not published within this article will be made available by request from any qualified investigator.

\section{Results}

The demographic and clinical characteristics of the studied sample are shown in Table 1 . There were no significant differences between patients in the two studied groups at baseline. Although the two groups showed a 
Table 1 Baseline characteristics of patients in the two studied groups

\begin{tabular}{lll}
\hline & Active $(n=14)$ & Control $(n=19)$ \\
\hline Age & $37.4(11.7)$ & $32.2(9.8)$ \\
Females & $13(92.9 \%)$ & 15 \\
Family history of migraine & $6(42.9 \%)$ & $8(42.1 \%)$ \\
Disease duration (years) & $8.5(6)$ & $7(4)$ \\
Migraine with aura & $2(14.3 \%)$ & $2(10.5 \%)$
\end{tabular}

Beck Depression Inventory

Mild

$$
9(64.3 \%)
$$

\begin{tabular}{lll} 
& \multicolumn{1}{c}{ Mild } & \multicolumn{1}{c}{$2(10.5 \%)$} \\
\multicolumn{1}{c}{ Borderline/moderate } & \multicolumn{1}{c}{$3(21.4 \%)$} & \\
Use of prophylaxis & $4(28.6 \%)$ & $6(31.6 \%)$ \\
Propranolol & 3 & 3 \\
Amitryptiline & 1 & 0 \\
Topiramate & 0 & 1 \\
Valproate Na & 0 & 1 \\
Cinnarizine & 0 & 1 \\
Migraine attack frequency & $8.2(2.4)$ & $7.3(2.9)$ \\
Migraine attack duration ${ }^{\mathrm{a}}$ & $15.8(16.4)$ & $19.3(20.4)$ \\
Pain intensity (NPRS) & $8.6(1.5)$ & $9.1(1.2)$ \\
Migraine days & 9.3 & $(1.9)$ \\
Severe migraine days & & $8.2(3.2)$ \\
HIT-6 & $7.2(1.8)$ & $70.6(8.1)$ \\
Severe HIT-6 (60 or more) & $73.2(5.7)$ & $14(73.7 \%)$ \\
Number of abortive pills & $15.9(11.8)$ & $13.8(9.5)$
\end{tabular}

Mean (SD) or patient numbers (percentage), NPRS Numeric Pain Rating Scale, HIT Headache Impact Test

aHours

${ }^{\mathrm{b}} \mathrm{NPRS}$ of 7

significant improvement in almost all the follow-up parameters, the magnitude of improvement was greater in the active treatment group (Table 2).

Regarding the inter-group comparisons, a 50\% reduction in migraine attacks and migraine days was achieved in $69.2 \%$ (9/13 cases) of the active treatment group versus a $25 \%(4 / 16)(p=0.02)$ and $31.2 \%$ reduction $(5 / 16)$ $(p=0.048)$ in the control group in both outcome measures, respectively. The severe migraine days were reduced by a minimum of $50 \%$ in $84.6 \%(11 / 13)$ cases in the active versus $50 \%(8 / 16)$ in the control group ( $p=$ 0.058) (Table 3).

Regarding the migraine attack characteristics, there was no significant difference between the two treatment groups. A 50\% reduction in the attack duration and intensity of pain was only achieved in four cases (3 active; 1 control) for both parameters. The absolute reduction in the duration of a single attack was similar between the two treatment groups (active 2.7(3.1) vs control 2.6(2.9)), while for NPRS, the active group was better (2.1(1.8), vs $1.3(1.2)$ for the control group). More cases in the active group (46.2\%, 6/ 13) achieved a $50 \%$ reduction in the number of abortive pills used when compared to the control group (31.2\%, 5/16). The difference was not statistically significant though $(p=0.3)$. Also, the mean decline in the number of pills was similar in both groups (active 6(5.1) vs control: 5.9(7.9)) (Table 3).

A clinically significant decrease in disability as measured by a 5 points reduction in HIT-6 score was achieved in $84.6 \%(11 / 13)$ of active cases vs $50 \% 8 / 16$ of controls $(p=0.058)$. The absolute HIT- 6 score reduction was $15.3(13.1)$ vs $6.1(5.2)$ in the active and control groups, respectively. The number of patients with severe HIT-6 score (60 or more) was reduced by $46.2 \%(6 / 13$ cases) in the active treatment group and by only $7.1 \%$ $(1 / 14$ cases $)$ in the control group $(p=0.02)$.

\section{Discussion}

Migraine is a chronic brain disease with an episodic clinical presentation [14]. The baseline hyperexcitability [15] is decreased by prophylactic treatment [16] and non-invasive brain stimulation [17], usually with a concomitant reduction in attack frequency. A recent study showed that a single pulse of TMS decreased firing in trigeminothalamic and thalamocortical

Table 2 Follow up of the two treatment groups

\begin{tabular}{|c|c|c|c|c|c|c|}
\hline & \multicolumn{3}{|l|}{ Active } & \multicolumn{3}{|l|}{ Control } \\
\hline & Baseline & Follow-up & $p$ value & Baseline & Follow-up & $p$ value \\
\hline Attack frequency & $8.2(2.4)$ & $5.2(3.5)$ & 0.001 & $7.3(2.9)$ & $5.8(2.7)$ & 0.025 \\
\hline Migraine days & $9.3(1.9)$ & $5.5(3.2)$ & 0.001 & $8.9(2.1)$ & $6.1(2.7)$ & 0.001 \\
\hline Severe migraine days & $7.2(1.8)$ & $1.5(1.9)$ & 0.001 & 8.2(3.2) & $4.9(3.2)$ & 0.02 \\
\hline Attack duration & 15.6(17.1) & $12.8(16)$ & 0.001 & $5.7(16.8)$ & $13.1(14.1)$ & 0.003 \\
\hline NPRS & $8.5(1.5)$ & $6.4(1.8)$ & 0.001 & $9(1.3)$ & $7.7(1.9)$ & 0.001 \\
\hline Abortive pills & $16.5(12.1)$ & $10.5(9.3)$ & 0.001 & $14.4(10.1)$ & $8.4(6)$ & 0.009 \\
\hline HIT-6 & $72.9(5.8)$ & $57.5(11)$ & 0.001 & 69.8(8.4) & 63.6(9.6) & 0.001 \\
\hline
\end{tabular}

Mean (SD), NPRS Numeric Pain Rating Scale, HIT Headache Impact Test 
Table 3 Comparison between the two treatment groups regarding the number of cases achieving a minimum of 50\% reduction, and the absolute reduction in different migraine severity parameters

\begin{tabular}{|c|c|c|c|}
\hline & Active $(n=13)$ [absolute reduction] & $\begin{array}{l}\text { Placebo }(n=16) \\
\text { [absolute reduction] }\end{array}$ & $p$ value \\
\hline Migraine attack frequency [number of attacks] & $9(69.2 \%)[3.1]$ & $4(25 \%)[1.5]$ & 0.02 \\
\hline Migraine days [number of days] & $9(69.2 \%)[3.8]$ & $5(31.2 \%)[2.8]$ & 0.048 \\
\hline Severe migraine days [number of days] & $11(84.6 \%)[5.7]$ & $8(50 \%)[3.3]$ & 0.058 \\
\hline NPRS [score difference] & $3(23 \%)[2.1]$ & $1(6.25 \%)[1.3]$ & 0.2 \\
\hline Single attack duration $[\mathrm{h}]$ & $3(23 \%)[2.7]$ & $1(6.25 \%)[2.6]$ & 0.19 \\
\hline Use of abortive pills [number of pills] & $6(46.2 \%)[6]$ & $5(32.2 \%)[5.9]$ & 0.3 \\
\hline
\end{tabular}

Patient numbers (percentage), NPRS Numeric Pain Rating Scale

neurons in rats after induction of cortical spreading depression [18]. When applied to the LDLPFC, rTMS exerted a bilateral non-opioid pain modulating effect [19-21]. In addition to changes in neuronal network activity, rTMS has been shown to induce changes in dopamine [22], and glutamate levels [23]. Repeated rTMS stimulation-induced synaptic plasticity via longterm potentiation, hence repeated sessions can induce a response that outlasts the stimulation period [24].

The main drugs used for episodic migraine prophylaxis include antiepileptics, like topiramate, and valproate, beta-blockers and amitriptyline [25]. Unfortunately, the effectiveness of these medications in reducing migraine frequency by at least $50 \%$ is in the range of $20-45 \%$, with a sham response rate of $10-25 \%$ and with $10-17 \%$ of cases stopping the treatments for intolerable side effects [4]. This leaves a big room for finding better management options for nonresponders that can achieve the main treatment pillars of $50 \%$ or more reduction in migraine frequency while reducing attack severity, and decreasing the need for abortive medications. This decreases the disability burden and also minimizes the risk of medication overuse or conversion to chronic migraine [26]. High-frequency rTMS applied over the LDLPFC was used by Brighina et al. in an open-label shamcontrolled study $(n=12)$ that recruited chronic migraineurs. The 6 cases in the active group achieved a significant reduction of migraine attacks $(>50 \%)$ [8]. Another study recruited 14 chronic migraine cases, who received 23 rTMS sessions over the LDLPFC on alternate days failed to show a significant improvement in the active treatment group [7]. Misra et al. applied three sessions of $10 \mathrm{~Hz}$ rTMS over the LDLPFC on alternate days in a double-blinded randomized sham-controlled RCT. They recruited a heterogenous group of 100 cases with chronic and episodic migraine, with or without aura, some of them were overusing abortive medications. The active treatment group improved greatly, with around $78.8 \%$ reduction in attack frequency in the active group vs a
33 reduction in the control group. The improvement was noticed mainly in the chronic migraine group [9]. A fourth RCT examined the effectiveness of rTMS applied to the primary motor area against an active comparator (Botulinum toxin) in chronic migraine. Their results showed a 50\% reduction of migraine days in $65-70 \%$ of cases in both treatment groups in the first month after therapy [27]. Low-frequency rTMS was tried over the vertex area in a shamcontrolled study that recruited 27 cases. Both groups showed a modest improvement but there was no difference between the two treatment groups [28].

Our study showed a reduction of $50 \%$ or more in migraine frequency and migraine days in almost $70 \%$ of the active treatment group. The sham response was $25 \%$. The absolute reduction in the number of attacks was 3.1 compared to a reduction of 2.4 attacks with topiramate $200 \mathrm{mg}$ [19].

Of note, although the sham stimulation group achieved a $25 \%$ reduction in attack frequency, only $1 / 14$ cases achieved a significant reduction in disability measured by HIT- 6 as opposed to 6/13 in the active group. This means that active LDLPFC rTMS helps more cases, especially severe ones, and contributes to disability reduction.

Regarding depressive symptoms, $91.9 \%$ of the studied sample had no or mild depression using the Beck Depression Inventory. However, due to the fact that LDLPFC high-frequency rTMS has a known antidepressant effect [29], it might be useful in future studies to correlate mood changes with the prophylactic role of rTMS in migraine.

Overall, rTMS is considered an attractive option for the prophylaxis of migraine. It is well tolerated and can be used independently or as an addition to pharmacological treatments [24]. Nevertheless, more work is needed at the bench side to better understand the correlation between the observed changes in neurotransmitter and neural activation studies in healthy and migraine cases, the clinical phenomenology of migraine, the rTMS stimulation parameters, 
and the clinical response to rTMS. This can help us to explain the discrepancies in clinical response to rTMS and to improve our selection process of cases who can benefit from this treatment.

\section{Abbreviations}

HIT 6: Headache Impact Test; ICHD: The international classfication of Headache disorder; LDLPFC: Left dorsolateral prefrontal cortex; MT: Motor threshold; NPRS: Numeric Pain Rating Scale; rTMS: Repetitive transcranial magnetic stimulation; SD: Standard deviation; SPSS: Statistical Package for Social Science

\section{Acknowledgements}

Not applicable

Ethics approval and consent to participate

The authors declare that the human experiments in this clinical trial have been approved by the ethical standard committee of Faculty of Medicine, AinShams University, on 19 July 2012 with reference number FMASU 1171/ 2012. The ethical committee of the Faculty of Medicine, AinShams University, has clearly approved experiments on human being (live vertebrates). All participants gave their written informed consent, and the study was approved by Ain Shams University ethical committee for a retrospective analysis that is IRB-approved. There is an approval of disclosure of all of the data that has been collected during the study and approved to be published into a journal or any public way. The study was a pilot clinical trial so did not need a registry number before the start of the trial. Trial Registration: ClinicalTrials.gov, Identifier: NCT04031781. Registered 23 July 2019-retrospectively registered at https://clinicaltrials.gov/ct2/show/NCT04031781?term=Migraine+Prophylaxis\&recrs=ce\&type $=$ Intr\&con $d=$ Migraine\&rank=9.

\section{Authors' contributions}

RA led and coordinated the data collection. TE led the team and was responsible for statistical analysis, revised and supervised the manuscript writing. SA led the coordination and communication between the team, study concept, and design. MH supervised the study and critical revision. NS reviewed the protocol and critical revision. SH study concept and design. SS and MS contributed to the acquisition of data, writing the manuscript, analysis, and interpretation. All authors read and approved the manuscript.

\section{Funding}

There has been no sponsorship for this study. Dr. Randa Amin, Dr. Tamer Emara, Dr. Samia Ashour, Dr. Mahmoud Hemeda, Dr. Nahed Salah El-Din, Dr. Salma Hamed, Dr. Sara Shouman, and Dr. Mohamed Shouman report no disclosure.

\section{Availability of data and materials}

Anonymized data of patients not published within this article will be made available by request from any investigator.

\section{Consent for publication}

Not applicable

\section{Competing interests}

The authors declare that they have no competing interests.

\section{Author details}

${ }^{1}$ Neurrology Department, Faculty of medicine, Ain Shams University, Abbasia, Cairo, Egypt. ${ }^{2}$ Resident of Family Medicine, Ain Shams University Hospitals, Abbasia, Cairo, Egypt. ${ }^{3}$ Resident of Toxicology, Ain Shams University Hospitals, Abbasia, Cairo, Egypt.

Received: 12 August 2019 Accepted: 19 December 2019 Published online: 03 February 2020

\section{References}

1. Vos T, Barber RM, Bell B, et al. Global, regional, and national incidence, prevalence, and years lived with disability for 301 acute and chronic diseases and injuries in 188 countries, 1990-2013: a systematic analysis for the Global Burden of Disease Study 2013. Lancet. 2015;386(9995):743-800.
2. Bigal ME, Serrano D, Buse D, Scher A, Stewart WF, Lipton RB. Acute migraine medications and evolution from episodic to chronic migraine: longitudinal population-based study. Headache. 2008;48(8):1157-68.

3. Bigal ME, Lipton RB. Modifiable risk factors for migraine progression. Headache. 2006;46(9):1334-43.

4. Shamliyan TA, Choi JY, Ramakrishnan R, et al. Preventive Pharmacologic Treatments for Episodic Migraine in Adults. J Gen Intern Med. 2013;28(9): 1225-37.

5. Lan L, Zhang X, Li X, Rong X, Peng Y. The efficacy of transcranial magnetic stimulation on migraine: a meta-analysis of randomized controlled trails. J Headache Pain. 2017;18:86.

6. Lipton RB, Dodick DW, Silberstein SD, et al. Single-pulse transcranial magnetic stimulation for acute treatment of migraine with aura: a randomised, doubleblind, parallel-group, sham-controlled trial. Lancet Neurol. 2010:9(4):373-80

7. Conforto AB, Amaro E, Gonçalves AL, et al. Randomized, proof-of-principle clinical trial of active transcranial magnetic stimulation in chronic migraine Cephalalgia. 2014;34(6):464-72.

8. Brighina F, Piazza A, Vitello $G$, et al. rTMS of the prefrontal cortex in the treatment of chronic migraine: a pilot study. J Neurol Sci. 2004;227(1): 67-71.

9. Misra UK, Kalita J, Bhoi SK. High-rate repetitive transcranial magnetic stimulation in migraine prophylaxis: a randomized, placebo-controlled study. J Neurol. 2013;260(11):2793-801.

10. Headache Classification Committee of the International Headache Society (IHS). The International Classification of Headache Disorders, 3rd edition (beta version). Cephalalgia. 2013;33(9):629-808.

11. Emara TH, Moustafa RR, ElNahas NM, et al. Repetitive transcranial magnetic stimulation at $1 \mathrm{~Hz}$ and $5 \mathrm{~Hz}$ produces sustained improvement in motor function and disability after ischaemic stroke. Eur J Neurol. 2010;17(9):12039.

12. Bayliss MS, Batenhorst AS. The HIT-6TM: a user's quide. Lincoln, RI: QualityMetric Incorporated, 2002.

13. Smelt AF, Assendelft WJ, Terwee CB, Ferrari MD, Blom JW. What is a clinically relevant change on the HIT-6 questionnaire? An estimation in a primary-care population of migraine patients. Cephalalgia 2014 Jan; 34(1):29-36. Miller S. The acute and preventative treatment of episodic migraine. Ann Indian Acad Neurol. 2012;15(Suppl 1):S33-9.

14. Haut SR, Bigal ME, Lipton RB. Chronic disorders with episodic manifestations: focus on epilepsy and migraine. Lancet Neurol. 2006;5:14857.

15. Goadsby PJ, Lipton RB, Ferrari MD. Migraine - current understanding and treatment. N Engl J Med. 2002;346:257-70.

16. Ayata C, Jin H, Kudo C, Dalkara T, Moskowitz MA. Suppression of cortical spreading depression in migraine prophylaxis. Ann Neurol. 2006:59:652-61.

17. Urora SK, Welch KM, Al-Sayed F. The threshold for phosphenes is lower in migraine. Cephalalgia. 2003;23:258-63.

18. Andreou AP, Holland PR, Akerman S, Summ O, Fredrick J, Goadsby PJ. Transcranial magnetic stimulation and potential cortical and trigeminothalamic mechanisms in migraine. Brain. 2016;139:2002-14.

19. Shehata HS, Esmail EH, Abdelalim A, et al. Repetitive transcranial magnetic stimulation versus botulinum toxin injection in chronic migraine prophylaxis: a pilot randomized trial. J Pain Res. 2016:9:771-7.

20. Lorenz J, Minoshima S, Casey KL. Keeping pain out of mind: the role of the dorsolateral prefrontal cortex in pain modulation. Brain. 2003;126(pt5):1079_ 91.

21. Jürgens TP, Leone M. Pearls and pitfalls: neurostimulation in headache. Cephalalgia. 2013;33(8):512-25

22. Strafella AP, Paus T, Barrett J, Dagher A. Repetitive transcranial magnetic stimulation of the human prefrontal cortex induces dopamine release in the caudate nucleus. J Neurosci. 2001;21:RC157.

23. Michael N, Gosling M, Reutemann $M$, et al. Metabolic changes after repetitive transcranial magnetic stimulation (rTMS) of the left prefrontal cortex. A sham-controlled proton magnetic resonance spectroscopy $(1 \mathrm{H}$ MRS) study of health brain. Eur J Neurosci. 2003;17:2462-8.

24. Lipton RB, Pearlman SH. Transcranial magnetic simulation in the treatment of migraine. Neurotherapeutics. 2010;7(2):204-12.

25. Estemalik E, Tepper S. Preventive treatment in migraine and the new US guidelines. Neuropsychiatr Dis Treat. 2013;9:709-20.

26. Saper JR, Diamond M, Couch JR, et al. Topiramate for migraine prevention: a randomized controlled trial. JAMA. 2004;291(8):965-73. 
27. Brighina F, De Tommaso M, Giglia F, et al. Modulation of pain perception by transcranial magnetic stimulation of left prefrontal cortex. J Headache Pain. 2011;12(2):185-91.

28. Teepker M, Hotzel J, Timmesfeld $\mathrm{N}$, et al. Low frequency rTMS of the vetex in the prophylactic treatment of migraine. Cephalalgia. 2010;30: $137-44$

29. Janicak PG, Dokucu ME. Transcranial magnetic stimulation for the treatment of major depression. Neuropsychiatr Dis Treat. 2015;11:1549-60.

\section{Publisher's Note}

Springer Nature remains neutral with regard to jurisdictional claims in published maps and institutional affiliations.

Submit your manuscript to a SpringerOpen ${ }^{\circ}$ journal and benefit from:

- Convenient online submission

- Rigorous peer review

- Open access: articles freely available online

High visibility within the field

- Retaining the copyright to your article

Submit your next manuscript at $\boldsymbol{\nabla}$ springeropen.com 